\title{
On an open problem in classification of languages
}

\author{
SANJAY JAIN \\ School of Computing \\ National University of Singapore \\ Singapore 119260 \\ sanjay@comp.nus.edu.sg
}

\begin{abstract}
Smith, Wiehagen and Zeugmann (1997) showed an interesting connection between learning with bounded number of mind changes from informants and classification from informant. They showed that if an indexed family of languages $\mathcal{L}$ is learnable via informants, using at most $m$ mind changes, then one can partition $2^{N}$, the class of all languages, into $m+2$ subclasses $\mathcal{L}_{1}, \ldots, \mathcal{L}_{m+2}$ such that $(1) \bigcup_{i \in\{1,2, \ldots, m+1\}} \mathcal{L}_{i}=\mathcal{L}$, and $(2)\left(\mathcal{L}_{1}, \ldots, \mathcal{L}_{m+2}\right)$ can be classified from informants. However Smith, Wiehagen and Zeugmann (1997) left open whether a similar result also holds for learning from texts. We show that such a result does not hold for texts.
\end{abstract}

\section{Introduction}

Consider the identification of formal languages from positive data. A learner is fed all the strings and no nonstrings of a language $L$ (the so called text of $L$ ), in any order, one string at a time. The learner, as it is receiving the data, outputs a sequence of grammars. The learner is said to identify (learn, infer) $L$ just in case the sequence of grammars converges to a grammar for $L$. A class of languages is learnable if some machine learns each language in the class. This is essentially the paradigm of identification in the limit (called TxtEx) introduced by Gold (1967). One may also consider the situation in the above model where a learner receives both positive and negative data, that is elements of the graph of the characteristic function of $L$ (called informant for $L$ ) as input. This leads to the identification criterion known as InfEx (see Gold (1967)).

Note that in the above learning model, a learner is expected to eventually come up with a grammar for the input language. Classification problem contrasts with the above model. Suppose we are given classes $\mathcal{L}_{1}, \mathcal{L}_{2}, \ldots, \mathcal{L}_{n}$. A classifier is given as input data about an arbitrary language $L \in \bigcup_{i \in\{1,2, \ldots, n\}} \mathcal{L}_{i}$. Based on the data, the classifier has to determine $i$ such that $L \in \mathcal{L}_{i}$. Thus, instead of eventually determining a grammar for $L$, the classifier just needs to say which class $L$ is from. Classification problem arises in several natural situations such as medical diagnosis. We refer the reader to Wiehagen and Smith (1995), and Smith, Wiehagen and Zeugmann (1997) for detailed discussion on the classification problem. Classification problem in different settings has also been studied by Duda and Hart (1973), Bylander, Allemang and Tanner (1991), Ben-David (1992), Cho and Reggia (1993), and Freivalds and Hoffmann (1994).

It is interesting to explore connections between identification of formal languages and classification. On one hand, one can find disjoint classes of languages which are finitely (that is 
without any mind changes) classifiable, but none of these classes is TxtEx or InfEx-identifiable. On the other hand, all infinite identifiable classes can be split into subclasses which are not classifiable, and into subclasses which are classifiable (where the classification machine is allowed to output arbitrarily on inputs from outside the class).

Smith, Wiehagen and Zeugmann (1997) showed an interesting connection between learning with bounded number of mind changes from informants and classification from informant. They showed that if an indexed family of languages $\mathcal{L}$ is learnable via informants, using at most $m$ mind changes, then one can partition $2^{N}$, the class of all languages, into $m+2$ subclasses $\mathcal{L}_{1}, \ldots, \mathcal{L}_{m+2}$ such that

(1) $\bigcup_{i \in\{1,2, \ldots, m+1\}} \mathcal{L}_{i}=\mathcal{L}$.

(2) $\left(\mathcal{L}_{1}, \ldots, \mathcal{L}_{m+2}\right)$ can be classified from informants.

Note that the above result is also related to learning by refutation (see Mukouchi and Arikawa (1993), Lange and Watson (1994), Jantke (1995), and Jain (1998)) where a machine is supposed to refute languages which are outside the class: the input language is outside $\mathcal{L}$ iff the classifier determines that the input language is in $\mathcal{L}_{m+2}$.

However Smith, Wiehagen and Zeugmann (1997) left open whether a similar result also holds for learning from texts. We show that such a result does not hold for texts. Thus, in some sense, relationship between identification and classification is much more complex when one considers learning from texts compared to when one considers learning from informant, even if one is restricting attention to limited number of mind changes.

We now proceed formally.

\section{Notations and identification criteria}

The recursion theoretic notions not explained below are from Rogers (1967). $N=\{0,1,2, \ldots\}$ is the set of all natural numbers, and this paper considers r.e. subsets $L$ of $N . \emptyset, \in, \subseteq, \supseteq, \subset, \supset$ denote empty set, member of, subset, superset, proper subset, and proper superset respectively. Cardinality of a set $S$ is denoted by $\operatorname{card}(S)$. We use card $(S) \leq *$ to denote that cardinality of set $S$ is finite. $2^{N}$ denotes the power set of $N,\{L \mid L \subseteq N\}$.

$\mathcal{R}$ denotes the set of total recursive functions from $N$ to $N$. $\mathcal{E}$ denotes the set of all recursively enumerable sets. $L$ ranges over $\mathcal{E}$. $\mathcal{L}$ ranges over subsets of $\mathcal{E}$. $\varphi$ denotes a standard acceptable programming system (acceptable numbering). $\varphi_{i}$ denotes the function computed by the $i$-th program in the programming system $\varphi$. We also call $i$ a program or index for $\varphi_{i}$. For a (partial) function $\eta$, domain $(\eta)$ and range $(\eta)$ respectively denote the domain and range of partial function $\eta$. $W_{i}$ denotes the domain of $\varphi_{i} . W_{i}$ is considered as the language enumerated by the $i$-th program in $\varphi$ system, and we say that $i$ is a grammar or index for $W_{i}$.

A text is a mapping from $N$ to $N \cup\{\#\}$. We let $T$ range over texts. Content of text $T$, content $(T)$, is defined to be the set of natural numbers in the range of $T$ (i.e. $\operatorname{content}(T)=$ range $(T)-\{\#\})$. $T$ is a text for $L$ iff $\operatorname{content}(T)=L$. That means a text for $L$ is an infinite sequence whose range, except for a possible \#, is just $L$.

An information sequence or informant is a mapping from $N$ to $(N \times N) \cup\{\#\}$. We let $I$ range over informants. Content of $I$, content $(I)$, is defined to be the set of pairs in the range of $I$ (i.e. content $(I)=\operatorname{range}(I)-\{\#\})$. An informant for $L$ is an infinite sequence $I$ such that $\operatorname{content}(I)=\{(x, 1) \mid x \in L\} \cup\{(x, 0) \mid x \notin L\}$. 
$\sigma$ and $\tau$ range over finite initial segments of texts or information sequences, where the context determines which is meant. $\Lambda$ denotes the empty sequence. We denote the set of finite initial segments of texts by SEG and set of finite initial segments of information sequences by SEQ. We use $\sigma \preceq T$ (respectively, $\sigma \preceq I, \sigma \preceq \tau$ ) to denote that $\sigma$ is an initial segment of $T$ (respectively, $I, \tau)$. $|\sigma|$ denotes the length of $\sigma . T[n]$ denotes the initial segment of $T$ of length $n$. Similarly, $I[n]$ denotes the initial segment of $I$ of length $n$.

A learning machine $\mathbf{M}$ is a mapping from initial segments of texts (information sequences) to $(N \cup\{?\})$. The point of using ?'s is to avoid biasing the count of mind changes by requiring a learning machine, on the empty sequence input, to output a program as its conjecture. For criteria of inference discussed in this paper we assume without loss of generality that, for all $\sigma \preceq \tau,[\mathbf{M}(\sigma) \neq ? \Rightarrow \mathbf{M}(\tau) \neq ?]$.

We say that $\mathbf{M}$ converges on $T$ to $i$, (written: $\mathbf{M}(T) \downarrow=i$ ) iff, for all but finitely many $n$, $\mathbf{M}(T[n])=i$. Convergence on information sequences is defined similarly.

Definition 1 (Gold (1967), Case and Smith (1983)) Suppose $a \in N \cup\{*\}$.

(a) $\mathbf{M} \mathbf{T x t E x} \mathbf{x}_{a}$-identifies $L$ (written: $L \in \mathbf{T x t E x}_{a}(\mathbf{M})$ ) iff, for all texts $T$ for $L$,

(i) $\mathbf{M}(T) \downarrow=j$ such that $W_{j}=L$.

(ii) $\operatorname{card}(\{n \mid ? \neq \mathbf{M}(T[n]) \neq \mathbf{M}(T[n+1])\}) \leq a$.

(b) $\mathbf{M} \mathbf{T x t E x}_{a}$-identifies $\mathcal{L}$ iff $\mathbf{M} \mathbf{T} \mathbf{x t E x} \mathbf{x}_{a}$-identifies each $L \in \mathcal{L}$.

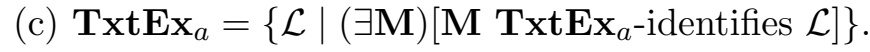

$\mathbf{T x t E x}_{*}$ is also referred to as $\mathbf{T x t E x}$. We call each instance of $? \neq \mathbf{M}(T[n]) \neq \mathbf{M}(T[n+1])$ as a mind change by $\mathbf{M}$ on $T$.

Definition 2 (Gold (1967), Case and Smith (1983)) Suppose $a \in N \cup\{*\}$.

(a) $\mathbf{M} \mathbf{I n f E x}_{a}$-identifies $L$ (written: $L \in \mathbf{I n f E x}_{a}(\mathbf{M})$ ) iff, for all information sequences $I$ for $L$,

(i) $\mathbf{M}(I) \downarrow=j$ such that $W_{j}=L$.

(ii) $\operatorname{card}(\{n \mid ? \neq \mathbf{M}(I[n]) \neq \mathbf{M}(I[n+1])\}) \leq a$.

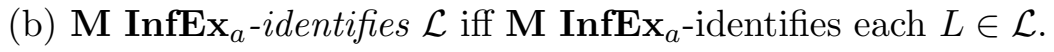

(c) $\operatorname{InfEx}_{a}=\left\{\mathcal{L} \mid(\exists \mathbf{M})\left[\mathbf{M} \operatorname{InfEx} \mathbf{x}_{a}\right.\right.$-identifies $\left.\left.\mathcal{L}\right]\right\}$.

A classifier is same as an inductive inference machine, except that its output is interpreted differently.

Definition 3 (Wiehagen and Smith (1995), Smith, Wiehagen and Zeugmann (1997)) Suppose $\mathcal{L}_{1}, \mathcal{L}_{2} \ldots, \mathcal{L}_{n} \subseteq 2^{N}$.

(a) $\mathbf{M}$ classifies $L$ from texts with respect to $\left(\mathcal{L}_{1}, \ldots, \mathcal{L}_{n}\right)$ iff, for all texts $T$ for $L, \mathbf{M}(T) \downarrow=j$ such that $L \in \mathcal{L}_{j}$.

(b) $\mathbf{M}$ classifies $\left(\mathcal{L}_{1}, \mathcal{L}_{2}, \ldots, \mathcal{L}_{n}\right)$ from texts iff $\mathbf{M}$ classifies each $L \in \bigcup_{i \in\{1,2, \ldots, n\}} \mathcal{L}_{i}$ from texts, with respect to $\left(\mathcal{L}_{1}, \mathcal{L}_{2}, \ldots, \mathcal{L}_{n}\right)$.

(c) $\mathbf{T x t C L}=\left\{\left(\mathcal{L}_{1}, \ldots, \mathcal{L}_{n}\right) \mid(\exists \mathbf{M})\left[\mathbf{M}\right.\right.$ classifies $\left(\mathcal{L}_{1}, \ldots, \mathcal{L}_{n}\right)$ from texts $\left.]\right\}$.

Definition 4 (Wiehagen and Smith (1995), Smith, Wiehagen and Zeugmann (1997)) Suppose $\mathcal{L}_{1}, \mathcal{L}_{2} \ldots, \mathcal{L}_{n} \subseteq 2^{N}$.

(a) M classifies $L$ from informants with respect to $\left(\mathcal{L}_{1}, \ldots, \mathcal{L}_{n}\right)$ iff, for all informants $I$ for $L$, $\mathbf{M}(I) \downarrow=j$ such that $L \in \mathcal{L}_{j}$. 
(b) $\mathbf{M}$ classifies $\left(\mathcal{L}_{1}, \mathcal{L}_{2}, \ldots, \mathcal{L}_{n}\right)$ from informants iff $\mathbf{M}$ classifies each $L \in \bigcup_{i \in\{1,2, \ldots, n\}} \mathcal{L}_{i}$ from informant, with respect to $\left(\mathcal{L}_{1}, \mathcal{L}_{2}, \ldots, \mathcal{L}_{n}\right)$.

(c) $\operatorname{InfCL}=\left\{\left(\mathcal{L}_{1}, \ldots, \mathcal{L}_{n}\right) \mid(\exists \mathbf{M})\left[\mathbf{M}\right.\right.$ classifies $\left(\mathcal{L}_{1}, \ldots, \mathcal{L}_{n}\right)$ from informants $\left.]\right\}$.

A class $\mathcal{L}=\left\{L_{0}, L_{1}, \ldots\right\}$ is said to be an indexed family iff there exists a recursive function $f$ such that, for all $i$ and $x,\left[x \in L_{i}\right.$ iff $\left.f(i, x)=1\right]$.

Smith, Wiehagen and Zeugmann (1997) showed that for any indexed family $\mathcal{L} \in \mathbf{I n f E x}_{m}$, there exists a partition of $2^{N}$ into $m+2$ disjoint classes $\mathcal{L}_{1}, \ldots, \mathcal{L}_{m+2}$ such that

(1) $\bigcup_{i \in\{1,2, \ldots, m+1\}} \mathcal{L}_{i}=\mathcal{L}$.

(2) $\left(\mathcal{L}_{1}, \ldots, \mathcal{L}_{m+2}\right) \in$ InfCL.

They however left open whether a similar result holds for learning/classification from texts. In this paper we show that this is not the case.

\section{Results}

We first show the following lemma, which is useful in proving our theorems. This lemma is essentially based on locking sequence argument (see Blum and Blum (1975)).

Lemma 5 Suppose $\left(\mathcal{L}_{1}, \mathcal{L}_{2}, \ldots, \mathcal{L}_{n}\right) \in \mathbf{T x t C L}$, where $\mathcal{L}_{i}$ 's are disjoint. Fix $i$ such that $1 \leq$ $i \leq n$. Suppose $L \in \mathcal{L}_{i}$. Then there exists a finite $S \subseteq L$ such that $\left(\forall L^{\prime} \mid S \subseteq L^{\prime} \subseteq L \wedge L^{\prime} \in\right.$ $\left.\bigcup_{j \in\{1,2, \ldots, n\}} \mathcal{L}_{j}\right)\left[L^{\prime} \in \mathcal{L}_{i}\right]$

Proof. Suppose $\left(\mathcal{L}_{1}, \mathcal{L}_{2}, \ldots, \mathcal{L}_{n}\right) \in \mathbf{T x t C L}$ as witnessed by $\mathbf{M}$. Let $L \in \mathcal{L}_{i}$ be given.

Claim 6 There exists a $\sigma$ such that $\operatorname{content}(\sigma) \subseteq L$, and

$$
(\forall \tau \mid \sigma \preceq \tau \wedge \operatorname{content}(\tau) \subseteq L)[\mathbf{M}(\tau)=\mathbf{M}(\sigma)]
$$

Proof. Suppose by way of contradiction otherwise. Thus

$$
(\forall \sigma \mid \operatorname{content}(\sigma) \subseteq L)(\exists \tau \mid \sigma \preceq \tau \wedge \operatorname{content}(\tau) \subseteq L)[\mathbf{M}(\sigma) \neq \mathbf{M}(\tau)]
$$

We will construct $\sigma_{j}, j \in N$, such that (i) content $\left(\sigma_{j}\right) \subseteq L$, (ii) $T=\bigcup_{j \in N} \sigma_{j}$ is a text for $L$, and (iii) $\mathbf{M}$ on $T$ makes infinitely many mind changes. This would be a contradiction to $\mathbf{M}$ witnessing that $\left(\mathcal{L}_{1}, \mathcal{L}_{2}, \ldots, \mathcal{L}_{n}\right) \in$ TxtCL.

Let $\sigma_{0}=\Lambda$. Suppose $\sigma_{j}$ has been defined. Let $\tau_{j}$ be such that $\sigma_{j} \preceq \tau_{j}$, $\operatorname{content}\left(\tau_{j}\right) \subseteq L$, and $\mathbf{M}\left(\sigma_{j}\right) \neq \mathbf{M}\left(\tau_{j}\right)$. Note that such a $\tau_{j}$ exists by (1). Let $\sigma_{j+1}$ be an extension of $\tau_{j}$ such that content $\left(\sigma_{j+1}\right)=\operatorname{content}\left(\tau_{j}\right) \cup\{x \mid x \in L \wedge x \leq j\}$.

It is easy to verify that (i), (ii) and (iii) above are satisfied. Claim follows.

Now by above claim there exists a $\sigma$, with content $(\sigma) \subseteq L$, such that

$$
(\forall \tau \mid \sigma \preceq \tau \wedge \operatorname{content}(\tau) \subseteq L)[\mathbf{M}(\tau)=\mathbf{M}(\sigma)] .
$$

Lemma follows by taking $S=\operatorname{content}(\sigma)$. 
Theorem 7 There exists an indexed family $\mathcal{L}$ such that

(a) $\mathcal{L} \in \mathbf{T x t E x}_{0}$ that

(b) for all $m \geq 1$, there does not exist a partition of $2^{N}$ into classes $\mathcal{L}_{1}, \mathcal{L}_{2}, \ldots, \mathcal{L}_{m+1}$ such

$$
\begin{aligned}
& \text { (b.1) } \mathcal{L}_{1} \cup \mathcal{L}_{2} \cup \cdots \cup \mathcal{L}_{m}=\mathcal{L} .\left(\text { Thus }, \mathcal{L}_{m+1}=2^{N}-\mathcal{L}\right) . \\
& \text { (b.2) }\left(\mathcal{L}_{1}, \mathcal{L}_{2}, \ldots, \mathcal{L}_{m+1}\right) \in \text { TxtCL. }
\end{aligned}
$$

Proof. Let $\mathcal{L}=\{N\}$ (i.e. $\mathcal{L}$ contains only one language, that is $N$ ). Clearly, $\mathcal{L} \in \mathbf{T x t E x}_{0}$. Now suppose by way of contradiction that, for some $m, \mathcal{L}_{1}, \mathcal{L}_{2}, \ldots, \mathcal{L}_{m+1}$, (b.1) and (b.2) above are satisfied. Without loss of generality, assume $m=1$ (since only one of $\mathcal{L}_{i}, 1 \leq i \leq m$, is nonempty). Thus, $\mathcal{L}_{1}=\mathcal{L}, \mathcal{L}_{2}=2^{N}-\{N\}$. Let $L=N$. Let $S$ be as given by Lemma 5 . Thus, all $L^{\prime} \supseteq S$ must belong to $\mathcal{L}_{1}=\mathcal{L}$, a contradiction to $\mathcal{L}_{1}=\mathcal{L}=\{N\}$. Thus, $\left(\mathcal{L}_{1}, \mathcal{L}_{2}\right) \notin$ TxtCL.

Theorem 7 can be extended as follows.

Theorem 8 For each $n \in N$, there exists an indexed family $\mathcal{L}$ of languages such that

(a) $\mathcal{L} \in \mathbf{T x t E x _ { n + 1 }}-\mathbf{T x t E x}_{n}$,

(b) for all $m$, there does not exist a partition of $2^{N}$ into classes $\mathcal{L}_{1}, \mathcal{L}_{2}, \ldots, \mathcal{L}_{m+1}$ such that

$$
\text { (b.1) } \mathcal{L}_{1} \cup \mathcal{L}_{2} \cup \cdots \cup \mathcal{L}_{m}=\mathcal{L} .\left(\text { Thus, } \mathcal{L}_{m+1}=2^{N}-\mathcal{L}\right) .
$$$$
\text { (b.2) }\left(\mathcal{L}_{1}, \mathcal{L}_{2}, \ldots, \mathcal{L}_{m+1}\right) \in \mathbf{T x t C L} .
$$

Proof. Let $L_{1}, L_{2}, \ldots, L_{n+2}$ be infinite recursive languages such that $L_{1} \subset L_{2} \subset \cdots \subset L_{n+2}$. Let $\mathcal{L}=\left\{L_{i} \mid 1 \leq i \leq n+2\right\}$.

Claim $9 \mathcal{L} \in \operatorname{TxtEx}_{n+1}-\operatorname{TxtEx}_{n}$.

Proof. We first show that $\mathcal{L} \in \mathbf{T} \mathbf{x t E x} \mathbf{x}_{n+1}$. Let $g_{i}$ denote a grammar for $L_{i}$. Define $\mathbf{M}$ as follows: $\mathbf{M}(\sigma)=g_{i}$, where $i$ is least such that content $(\sigma) \subseteq L_{i}$. It is easy to verify that $\mathbf{M}$ $\operatorname{TxtEx}_{n+1}$-identifies $\mathcal{L}$.

Now suppose by way of contradiction that some $\mathbf{M} \mathbf{T} \mathbf{T t E x} \mathbf{x}_{n}$-identifies $\mathcal{L}$. Then define $\sigma_{i}$, $i \in\{1,2, \ldots, n+2\}$, as follows.

Let $\sigma_{1}$ be such that content $\left(\sigma_{1}\right) \subseteq L_{1}$, and $\mathbf{M}\left(\sigma_{1}\right)$ is a grammar for $L_{1}$. Note that there must exist such a $\sigma_{1}$, since $\mathbf{M}$ TxtEx-identifies $L_{1}$. Now suppose $\sigma_{i}$ has been defined (here $1 \leq i \leq n+1)$. Then define $\sigma_{i+1}$ as follows: $\sigma_{i+1}$ is an extension of $\sigma_{i}$ such that $\operatorname{content}\left(\sigma_{i+1}\right) \subseteq$ $L_{i+1}$, and $\mathbf{M}\left(\sigma_{i+1}\right)$ is a grammar for $L_{i+1}$. Note that there must exist such a $\sigma_{i+1}$, since $\mathbf{M}$ TxtEx-identifies $L_{i+1}$. Now let $T$ be an extension of $\sigma_{n+2}$ such that $T$ is a text for $L_{n+2}$. Now

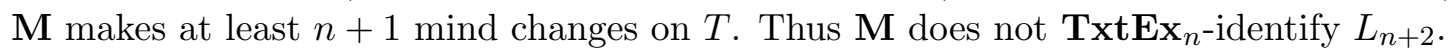

Now suppose by way of contradiction that, for some $m, \mathcal{L}_{1}, \mathcal{L}_{2}, \ldots, \mathcal{L}_{m+1}$, (b.1) and (b.2) above are satisfied. Let $L=L_{1}$. Without loss of generality suppose $L \in \mathcal{L}_{1}$, and let $S$ be as given by Lemma 5 for $L$. Thus, for all $L^{\prime}$ such that $S \subseteq L^{\prime} \subseteq L_{1}, L^{\prime} \in \mathcal{L}_{1}$. However, this is impossible since there are infinitely many such $L^{\prime}$, but $\mathcal{L}_{1}$ is finite (since $\mathcal{L}$ is finite). Thus, $\left(\mathcal{L}_{1}, \mathcal{L}_{2}, \ldots, \mathcal{L}_{m+1}\right) \notin$ TxtCL. 
Corollary 10 For each $m$, there exists an indexed family $\mathcal{L}$ of languages such that

(a) $\mathcal{L} \in \mathbf{T x t E x}_{m}$,

(b) $\mathcal{L} \notin \mathbf{T x t E x _ { n }}$, for $n<m$.

(c) there does not exist a partition of $2^{N}$ into classes $\mathcal{L}_{1}, \mathcal{L}_{2}, \ldots, \mathcal{L}_{m+2}$ such that

(c.1) $\mathcal{L}_{1} \cup \mathcal{L}_{2} \cup \cdots \cup \mathcal{L}_{m+1}=\mathcal{L}$. (Thus, $\left.\mathcal{L}_{m+2}=2^{N}-\mathcal{L}\right)$.

(c.2) $\left(\mathcal{L}_{1}, \mathcal{L}_{2}, \ldots, \mathcal{L}_{m+2}\right) \in$ TxtCL.

Note that the diagonalizing classes in above theorems and corollary can be easily made infinite by changing $\mathcal{L}$ to $\mathcal{L}^{\prime}$ as follows. For $L \in \mathcal{L}$, let $L_{i}^{\prime}=\{\langle i, x\rangle \mid x \in L\}$. Let $\mathcal{L}^{\prime}=\left\{L_{i}^{\prime} \mid L \in\right.$ $\mathcal{L}, i \in N\}$.

\section{Acknowledgements}

The author was supported in part by NUS grant number RP3992710.

\section{References}

Ben-David, S. (1992). Can finite samples detect singularities of real-valued functions? In Symposium on the Theory of Computation, (pp. 390-399).

Blum, L. and Blum, M. (1975). Toward a mathematical theory of inductive inference. Information and Control, 28, 125-155.

Bylander, T., Allemang, D., and Tanner, M. (1991). The computational complexity of diagnosis. Artificial Intelligence, 49, 25-60.

Case, J. and Smith, C. (1983). Comparison of identification criteria for machine inductive inference. Theoretical Computer Science, 25, 193-220.

Cho, S. and Reggia, J. (1993). Multiple disorder diagnosis with adaptive competitive neural networks. Artificial Intelligence in Medicine, 5, 469-487.

Duda, R. and Hart, P. (1973). Pattern Classification and Scene Analysis. Wiley-Interscience Publication.

Freivalds, R. and Hoffmann, A. G. (1994). An inductive inference approach to classification. Journal of Experimental and Theoretical Artificial Intelligence, 6, 63-72.

Gold, E. M. (1967). Language identification in the limit. Information and Control, 10, 447-474.

Jain, S. (1998). Learning with refutation. Journal of Computer and System Sciences, 57(3), $356-365$.

Jantke, K. P. (1995). Reflecting and self-confident inductive inference machines. In Jantke, K., Shinohara, T., and Zeugmann, T. (Eds.), Algorithmic Learning Theory: Sixth International Workshop (ALT '95), volume 997 of Lecture Notes in Artificial Intelligence, (pp. 282-297). Springer-Verlag. 
Lange, S. and Watson, P. (1994). Machine discovery in the presence of incomplete or ambiguous data. In Arikawa, S. and Jantke, K. (Eds.), Algorithmic Learning Theory: Fourth International Workshop on Analogical and Inductive Inference (AII '94) and Fifth International Workshop on Algorithmic Learning Theory (ALT '94), volume 872 of Lecture Notes in Artificial Intelligence, (pp. 438-452). Springer-Verlag.

Mukouchi, Y. and Arikawa, S. (1993). Inductive inference machines that can refute hypothesis spaces. In Jantke, K., Kobayashi, S., Tomita, E., and Yokomori, T. (Eds.), Algorithmic Learning Theory: Fourth International Workshop (ALT'93), volume 744 of Lecture Notes in Artificial Intelligence, (pp. 123-136). Springer-Verlag.

Rogers, H. (1967). Theory of Recursive Functions and Effective Computability. McGraw-Hill. Reprinted by MIT Press in 1987.

Smith, C., Wiehagen, R., and Zeugmann, T. (1997). Classifying predicates and languages. International Journal of Foundations of Computer Science, 8, 15-41.

Wiehagen, R. and Smith, C. H. (1995). Generalization versus classification. Journal of Experimental and Theoretical Artificial Intelligence, 7, 163-174. 